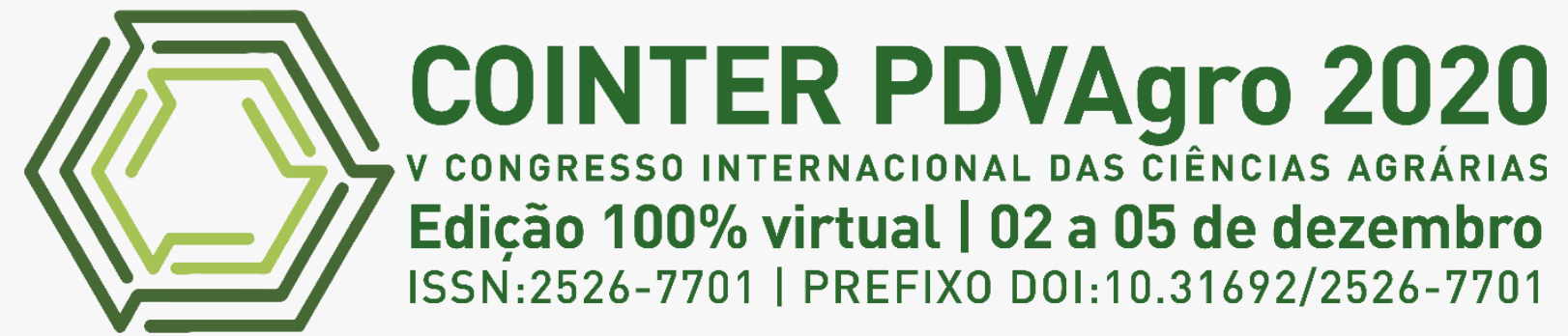

\title{
VIABILIDADE DA UTILIZAÇÃO DA MANDIOCA PARA PRODUÇÃO DE ETANOL
}

\section{VIABILIDAD DEL USO DE YUCA PARA LA PRODUCCIÓN DE ETANOL}

\section{FEASIBILITY OF THE USE OF CASSAVA FOR ETHANOL PRODUCTION}

\author{
Apresentação: Comunicação Oral \\ Ester dos Santos Coêlho하 ; Elidayane da Nóbrega Santos²; Edilayane da Nóbrega Santos ${ }^{3}$; Aurélio Paes Barros \\ Júnior $^{4}$; Lindomar Maria da Silveira ${ }^{5}$
}

DOI: https://doi.org/10.31692/2526-7701.VCOINTERPDVAgro.0534

\begin{abstract}
RESUMO
A mandioca pode ser utilizada para diversos fins e pode-se utilizar todas as partes da planta, sendo os tubérculos consumidos in natura ou destinados para indústria alimentícia para produção de farinha e fécula. Este estudo objetivou realizar uma busca bibliográfica sobre a viabilidade da utilização de mandioca para produção de etanol, mediante trabalhos ciêntifcos publicados, monografias, disseratções e teses. Este trabalho é uma pesquisa exploratória de natureza qualitativa, que utilizou dados de trabalhos científicos publicados em periódicos nacionais e internacionais, bem como dissertações e teses, realizando as buscas de forma digital utilizando o Google acadêmico, Science Direct e SciELO. De acordo com a revisão de literatura, pode-se perceber que a cultura da mandioca apresenta grande potencialidade para a obtenção do etanol, por armazenar amido em suas raízes a mandioca requer processos para poder ser realizada a fermentação alcoólica. Ainda há um amplo espaço para ser explorado em termos de produtividade para a cultura da mandioca, enquanto que para a cana-de-açúcar que é a matéria-prima mais utilizada, os custos de produção estão cada vez maiores e com menores incrementos de produtividade. Além da sua alta potencialidade, o cultivo da mandioca para fins de produção de biocombustíveis apresenta vantagens em virtude do seu fácil cultivo, podendo ser cultivada em áreas que apresentam improdutividade para as demais culturas, com poucas exigências hídricas e nutricionais. Assim, que haja uma expansão e melhor aproveitamento da mandioca como matéria-prima para a produção do etanol, é necessário a realização de pesquisas que ofereçam subsídios para a redução os custos de produção, bem como maximizem a produtividade da mandioca.
\end{abstract}

Palavras-chave: amido, biocombustível, Manihot esculenta Crantz.

\section{RESUMEN}

La yuca se puede utilizar para diversos fines y se pueden utilizar todas las partes de la planta, consumiéndose los tubérculos frescos o destinados a la industria alimentaria para la producción de harina

\footnotetext{
${ }^{1}$ Mestranda em Fitotecnia, Universidade Federal Rural do Semi-Árido (UFERSA), estersantos12@hotmail.com

${ }^{2}$ Mestranda em Fitotecnia, UFERSA, dayane-nobrega@ outlook.com

${ }^{3}$ Doutoranda em Ciência dos Alimentos, Universidade Federal de Lavras (UFLA), layane.nobrega@hotmail.com

${ }^{4}$ Doutor em Agronomia, UFERSA, aurelio.barros@ufersa.edu.br

${ }^{5}$ Doutor em Agronomia, UFERSA, lindomarmaria@ufersa.edu.br
} 
y almidón. Este estudio tuvo como objetivo realizar una búsqueda bibliográfica sobre la viabilidad del uso de la yuca para la producción de etanol, a través de trabajos científicos publicados, monografías, disertaciones y tesis. Este trabajo es una investigación exploratoria de carácter cualitativo, que utilizó datos de trabajos científicos publicados en revistas nacionales e internacionales, así como disertaciones y tesis, realizando búsquedas de forma digital utilizando revistas académicas de Google, Science Direct y SciELO. De acuerdo con la revisión de la literatura, se puede observar que el cultivo de yuca tiene un gran potencial para la obtención de etanol, el almacenamiento de almidón en sus raíces requiere procesos de yuca para poder realizar la fermentación alcohólica. Aún queda mucho espacio por explorar en términos de productividad para el cultivo de yuca, mientras que para la caña de azúcar, que es la materia prima más utilizada, los costos de producción son cada vez más altos y con menores incrementos de productividad. Además de su alto potencial, el cultivo de yuca con fines de producción de biocombustible tiene ventajas debido a su fácil cultivo, que se puede cultivar en áreas improductivas para otros cultivos, con pocos requerimientos hídricos y nutricionales. Así, si existe una expansión y mejor uso de la yuca como materia prima para la producción de etanol, es necesario realizar una investigación que ofrezca subsidios para reducir los costos de producción, así como maximizar la productividad de la yuca.

Palabras Clave: almidón, biocombustible, Manihot esculenta Crantz.

\begin{abstract}
Cassava can be used for various purposes and all parts of the plant can be used, with the tubers being consumed fresh or destined for the food industry for the production of flour and starch. This study aimed to carry out a bibliographic search on the viability of using cassava for ethanol production, through published scientific works, monographs, dissertations and theses. This work is an exploratory research of a qualitative nature, which used data from scientific works published in national and international journals, as well as dissertations and theses, performing searches in a digital way using Google academic, Science Direct and SciELO. According to the literature review, it can be seen that the culture of cassava has great potential for obtaining ethanol, storing starch in its roots requires cassava processes to be able to carry out alcoholic fermentation. There is still ample space to be explored in terms of productivity for the cassava crop, while for sugarcane, which is the most used raw material, production costs are increasingly higher and with lower productivity increases. . In addition to its high potential, the cultivation of cassava for the purpose of biofuel production has advantages due to its easy cultivation, which can be grown in areas that are unproductive for other crops, with few water and nutritional requirements. Thus, if there is an expansion and better use of cassava as a raw material for ethanol production, it is necessary to carry out research that offers subsidies to reduce production costs, as well as maximizing cassava productivity.
\end{abstract}

Keywords: biofuel, Manihot esculenta Crantz, , starch.

\title{
INTRODUÇÃO
}

A mandioca (Manihot esculenta Crantz), é uma planta da classe das dicotiledonêas, ordem Euphorbiales, pertencente à família Euphorbiaceae e ao gênero Manihot (ORLANDINNI; LIMA, 2014). A espécie teve sua origem difundida na América Tropical, atualmente é mundialmente consumida por diversas pessoas, e considerada a única espécie alimentícia abrangente por esse gênero (IYER et al., 2010).

A cultura apresenta baixo custo de produção e facilidade no plantio, podendo adaptarse a diversas condições edafoclimáticas e apresentar resistência a insetos, sendo considerada uma cultura rústica alimentícia (FIORDA et al., 2013; MARTINEZ; FEIDEN, 2017). A mandioca é de ampla importância para o agronegócio no mundo, gerando emprego em várias áreas rurais em inúmeros países, com uma produção mundial de aproximadamente 292 milhões 
de toneladas (FAOSTAT, 2019). É considerada uma cultura polivalente e participa de programas que buscam intensificar a sustentabilidade na produção, combatendo a fome a partir do manejo ideal do plantio, do solo e da água (FAO 2013).

Diante das demandas do mercado cada vez mais competitivas, as mudanças econômicas induziram o setor a reexaminar seus processos e a qualidade do produto, em que os resíduos gerados pela cultura da mandioca começam a ser vistos como renda potencial (MARTINEZ; FEIDEN, 2017). Tendo em vista esse aspecto, os resíduos obtidos a partir do processamento das raízes da mandioca podem ser líquidos ou sólidos. O principal resíduo líquido é a manipueira, e dentre os resíduos sólidos têm-se a fibra, a varredura, a casca e entrecasca, o farelo (MARTINEZ; FEIDEN, 2017). Esses resíduos apresentam aproximadamente 26,8\% de fibra, $58,1 \%$ de amido, $88 \%$ de matéria seca e 3,4\% de proteína bruta, indicando assim um bom conteúdo de amido e qualidade de fibra (LACERDA et al., 2009). Portanto, visando maximizar a utilização da mandioca, esses resíduos podem ser transformados em produtos de alto valor (ZHANG et al., 2006).

A matéria prima produzida a partir da mandioca apresenta características competitivas, semelhantes ao milho e arroz, sendo uma alternativa para substituição desses alimentos servindo como fonte para a formulação de biodegradáveis, como os produtos derivados do petróleo, a exemplo do plástico e etanol (VALLE; LORENZI, 2014). Este estudo objetivou realizar uma busca bibliográfica sobre a viabilidade da utilização de mandioca para produção de etanol, mediante trabalhos ciêntifcos publicados, monografias, disseratções e teses.

\section{FUNDAMENTAÇÃO TEÓRICA}

\section{Importância Socioeconômica da mandioca}

Segundo dados da FAOSTAT (2019), dentre os maiores produtores mundiais da mandioca o Brasil se encontra na quarta posição, atrás de Nigéria, Tailândia e Indonésia. Constitui-se como a terceira maior fonte alimentícia em regiões tropicais, após a cultura do milho e do arroz. Os maiores produtores no Brasil encontram-se no Estado do Paraná correspondendo a 70,1\% da população nacional, no Estado do Mato Grosso do Sul com 20,3\% e São Paulo com 8,7\%. Segundo a Organização das Nações Unidas para a Alimentação e Agricultura (FAO), no ano de 2018, o Brasil produziu cerca de 18 milhões de toneladas de mandioca (FAO, 2020).

O sucesso da mandioca pode ser atribuído as suas múltiplas aplicações, podendo ser processada e comercializada como farinhas, biscoitos, biocombustíveis, utilizados também como fonte de proteína em rações animais (ANDRÉ; SANTOS, 2012). Um dos fatores 
VIABILIDADE DA UTILIZAÇÃO DA MANDIOCA PARA PRODUÇÃO DE ETANOL

determinantes no direcionamento do uso da mandioca é o teor de ácido cianídrico, sendo classificadas como mandioca mansa aquelas com menos de $50 \mathrm{mg}$ de $\mathrm{HCN}$ por quilo de raiz fresca, ou mandioca brava aquelas com mais de $100 \mathrm{mg}$ de $\mathrm{HCN}$ por quilo de raiz fresca (SILVA, 2018).

A mandioca pode ser utilizada para diversos fins e pode-se utilizar todas as partes da planta, sendo os tubérculos consumidos in natura ou destinados para indústria alimentícia para produção de farinha e fécula. A parte área da planta é utilizada como forragem, servido de alimentos para os animais em vários períodos do ano. Durante períodos críticos, a viabilidade econômica da cultura da mandioca para a pecuária pode aumentar, devido ao alto valor nutritivo, sendo de boa digestibilidade e aceitação pelos animais (FERREIRA et al., 2009).

Além da importância em âmbito mundial, a expansão regional da mandioca deve-se a agricultura familiar e ao pequeno produtor. Desta forma, é imprescindível o acesso à políticas agrícolas de incentivo ao complexo agroindustrial nacional e o desenvolvimento e estabelecimento de uma ampla cadeia produtiva (MDA, 2016).

\section{Importância do biocombustível}

Os biocombustíveis são combustíveis obtidos por meio da biomassa, podendo ser classificado como biocombustíveis de primeira geração, quando a finalidade do cultivo é para fins energéticos ou biocombustíveis de segunda geração, quando se obtém por meio dos resíduos de outros processos realizados na matéria-prima (CINELLI, 2012). A importância destes dá-se devido a redução da emissão dos gases do efeito estufa, fornecendo assim uma energia de segurança, e contribuindo para o desenvolvimento socioeconômico em áreas rurais, considerando a forma de condução para obtenção do biocombustível (TAYLOR et al., 2009), uma vez que a utilização inadequada dos insumos para obtenção dos biocombustíveis também ocasionam problemas, pois a queima dos biocombustíveis pode elevar as emissões de produtos com grande toxidez (BIOFUEL, 2019), além disso deve-se considerar o impacto ambiental gerado por meio dos cultivos das plantas que fornecem a biomassa, como uso incorreto de água, defensivos e fertilizantes.

Objetivando reduzir o consumo de combustíveis fósseis, o etanol constitui-se como uma fonte de energia alternativa dentre os biocombustíveis (ARREDONDO, 2009). A obtenção do etanol pode ser por meio sintético ou pela fermentação. No Brasil, devido sua ampla disponibilidade de matérias-primas, a fermentativa é mais utilizada (LIMA, 2017). Buscando a inovação e exploração de resíduos de biomassa enfatizando uma visão de sustentabilidade, algumas matérias-primas vêm sendo analisadas, no entanto alguns fatores devem ser levados 
em consideração para a escolha da matéria-prima ideal, como por exemplo o seu preço e disponibilidade, tecnologia disponível, mercado dos insumos, bem como a sua qualidade após o processo de conversão e a promoção da sustentabilidade (OLIVEIRA et al., 2012; LIMA, 2017).

O biocombustível gerado a partir da biomassa lignocelulósica tem se estabelecido como uma alternativa viável para a obtenção de combustíveis (SILVA, 2012; LOUREIRO et al.,2020). A composição da biomassa constitui-se em lignina, celulose, extrativos, hemicelulose, cinzas e outros compostos com menor relevância e proporção, sendo a celulose e hemicelulose 70\% de todo conteúdo da biomassa (LIMA, 2013; LOUREIRO et al., 2020). Para que o conteúdo de celulose e hemicelulose seja disponibilizado na forma de açúcares fermentescíveis, é necessário a realização de um pré-tratamento desses compostos (DIAS et al., 2013).

Instituída pela Lei 13.576, de 26 de dezembro de 2017, a Política Nacional de Biocombustíveis (RenovaBio), foi implementada com a meta de possibilitar o aumento da produção de biocombustíveis no País em condições sustentáveis. As vantagens e a importância do uso dos biocombustíveis basicamente é que eles são biodegradáveis, e apresentam um baixo teor de enxofre e compostos aromáticos, com isso causam menores impactos ao meio ambiente do que os combustíveis fósseis (ANP, 2019).

\section{Etanol}

O etanol constitui-se como o principal biocombustível utilizado em todo o mundo, representando $10 \%$ da energia mundial, e algumas previsões futuras estimam que o uso mundial do etanol atingirá aproximadamente $27 \%$ até 2050 (IEA, 2017). A produção mundial de etanol atinge em torno de 80 bilhões de litros por ano, e a maior parte desta produção (cerca de 95\%) dá-se pela fermentação de determinados açúcares pela levedura Saccharomyces cerevisiae (RFA, 2016). Essa produção mundial concentra-se principalmente nos EUA (52\%) e no Brasil (25\%), nesse contexto a nível mundial a matéria-prima mais utilizada é o milho (67\%), seguido pela cana-de-açúcar (OCDE, 2016). Destaca-se também no mercado mundial do etanol o país da China.

O etanol constitui-se como o biocombustível mais usado no mundo, com rápida expansão de uso global, uma vez que seu uso é bastante difundido (CINELLI, 2012). Para a produção do etanol há uma diversidade de matérias-primas que podem ser aplicadas a essa finalidade, estas se dividem nas seguintes categorias: os sacaríneos que englobam alguns produtos como cana-de-açúcar, beterraba, sorgo sacarino; os amiláceos como por exemplo a 
mandioca, batata, sorgo granífero e cereais; e por fim a categoria dos materiais celulósicos, tais como o marmeleiro, o eucalipto, sabugo de milho, casca de arroz e bagaço de cana (OLIVEIRA et al., 2012).

Diante desses aspectos de diversidade de matérias-primas, na Alemanha a obtenção do etanol baseia-se no uso de cereais e da beterraba, enquanto que na França é baseada em milho, trigo e beterraba. A produção do etanol no Canadá é principalmente por meio de grãos, semelhante a China que obtém com o uso de grãos e tubérculos (mandioca). No Brasil a matériaprima mais utilizada é cana-de-açúcar, devido sua tradição (SORDA et al., 2010; USDA, 2011).

\section{Procedimentos tecnológicos para produção do etanol de mandioca}

O amido é uma fonte de carboidrato utilizado para diversos processos de fermentação, a exemplo da produção de etanol. Porém este carboidrato não pode ser assimilado diretamente pelas leveduras, necessitando-se de uma hidrólise e sacarificação para obter a glicose, maltose e outros compostos assimiláveis pelos microrganismos (SILVA, 2009). Estudos confirmam que o amido obtido a partir da mandioca possui quantidade menor de matérias graxas em comparação ao extraído do milho e batata doce (CAMPOS et al., 2006; ZAMORAHERNÁNDEZ et al., 2014). Por outro lado, o amido hidrolisado da mandioca apresenta alta concentração de carboidrato, necessitando ser aditivado com outros compostos para a preparação do inóculo, como carbono e nitrogênio para se adequar a fermentação alcoólica (ZAMORA-HERNÁNDEZ et al., 2014).

A via fermentativa é o método mais empregado para obter o etanol de primeira geração, por meio da fonte amilácea como exposto na Figura 1.

Figura 01. Rotas tecnológicas para produção de etanol de fonte amilácea

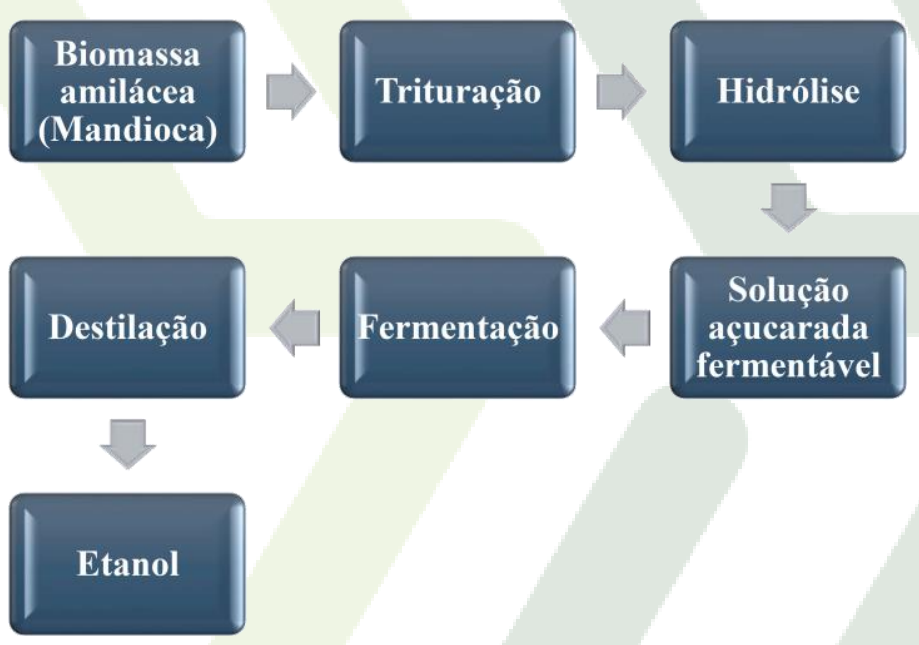

Fonte: Adaptado de Martinez (2016). 
As fontes amiláceas extraídas do amido são alternativas para a produção de biocombustível, a exemplo do etanol. Portanto, necessita-se que a matéria prima seja processada por hidrólise para converter o amido em açúcares fermentáveis. Esse processo de hidrólise pode ser realizado por duas etapas: hidrólise enzimática e hidrólise ácida (SUMAN et al., 2011).

\section{Hidrólise enzimática}

A amilose e amilopectinas são os dois polissacarídeos que compõem o amido, e suas proporção variam de acordo com a espécie e grau de maturação de cada planta (BOBBIO; RIBEIRO, 2011). A hidrólise enzimática também é conhecida como sacarificação, nessa etapa ocorre degradação dos polissacarídeos que constituem o amido ou os materiais lignocelulósicos transformando-os em açúcares para as etapas seguintes (PEREIRA et al., 2016). Quando se utiliza matérias lignocelulósicos da mandioca para a produção do etanol a conversão da biomassa é por meio de enzimas celulolíticas (ASGHER et al., 2013; IQBAL et al., 2013).

De acordo com Shahzadi et al. (2014), a hidrólise da celulose encontrada nos materiais lignocelulósicos têm se apresentado como uma alternativa viável e promissora para a produção do etanol. Para o uso dessas enzimas não são requeridos custos elevados em equipamentos, pois possuem grande especificidade (RABELO et al., 2011). Para a hidrólise do amido são requeridas enzimas amilolíticas, que podem ser encontradas em plantas, animais e de forma mais abundante em microorganismos (ARAÚJO, 2012). A etapa da hidrólise enzimática do amido é necessária devido a ausência das enzimas amilolíticas nos microorganismos que agem na fermentação (RIBEIRO, 2011). Basicamente, as enzimas mais utilizadas são $\alpha$ amilases, $\beta$ amilases e amiloglucosidase (ARAÚJO, 2012). As $\alpha$ amilases apresentam uma grande termoestabilidade, e essa característica ocasiona o aumento do custo na unidade operacional, para isso há uma busca por meio da biotecnologia para produzir $\alpha$ amilases termoestáveis e consequentemente com um custo mais baixo (CUNHA et al., 2009).

Os parâmetros para a boa atuação das amilases encontram-se em $\mathrm{pH}$ de 4-7 e temperaturas de $30-130^{\circ} \mathrm{C}$ (PANDEY, et al., 2005). Além das amilases, existem outras enzimas utilizadas na hidrólise do amido (Figura 2). 
VIABILIDADE DA UTILIZAÇÃO DA MANDIOCA PARA PRODUÇÃO DE ETANOL

Figura 02. Exemplos de enzimas envolvidas na hidrólise do amido

\begin{tabular}{|c|c|c|c|c|c|}
\hline \multirow{2}{*}{ Tipo } & \multirow{2}{*}{ Nome comum } & \multirow{2}{*}{$\begin{array}{c}\text { Microrganismos } \\
\text { produtores }\end{array}$} & \multirow{2}{*}{ Substrato } & \multicolumn{2}{|c|}{ Ótimo } \\
\hline & & & & $\mathrm{pH}$ & $\mathrm{T}^{\circ} \mathrm{C}$ \\
\hline \multirow[t]{4}{*}{ Endoamilase } & Amilase & B. subtilis & $\alpha$-1,4-glicosil & 6,0 & $\begin{array}{l}65- \\
70\end{array}$ \\
\hline & bacteriana & B. licheniformis & $\alpha$-1,4-glicosil & 5,0 & 90 \\
\hline & & & & 7,0 & \\
\hline & & A.Oryzae & $\alpha$-1,4-glicosil & 4,5 & $\begin{array}{l}50- \\
60\end{array}$ \\
\hline \multirow[t]{5}{*}{ Exoamilase } & Amilase fúngica & A. Niger & $\alpha$-1,4-glicosil & $4.0-6.0$ & 60 \\
\hline & Amiloglucosidade & & $\alpha$-1,6-glicosil & & \\
\hline & & Bacillus sp & $\alpha-1,4$-glicosil & 5.0 & $\begin{array}{l}55- \\
60\end{array}$ \\
\hline & $\alpha$-amilase & Clostridium $s p$ & $\alpha-1,4$-glicosil & $5.5-6.0$ & $\begin{array}{l}75- \\
85\end{array}$ \\
\hline & Bacteriana & & & & \\
\hline \multirow[t]{2}{*}{$\alpha-1,6$ amilase } & Pululanase & K. aerogenes & $\begin{array}{l}\alpha-1,6- \\
\text { Maltotriosil }\end{array}$ & 5.0 & 60 \\
\hline & Isoamilase & Pseudomonas sp. & $\alpha-1,6-$ Heptasac & 4.0 & $\begin{array}{l}50- \\
55\end{array}$ \\
\hline Isomerase & Glicose isomerase & A. Circulans & $\begin{array}{l}\text { Aldo/ceto } \\
\text { pentose } \\
\text { Aldo/ceto } \\
\text { hexose }\end{array}$ & 8.2 & 65 \\
\hline
\end{tabular}

Fonte: Araújo, (2012); adaptado de Fenemma (2010).

A hidrólise enzimática do amido inicia com a quebra parcial das ligações glicosídicas, com a produção de açúcares $(15-30 \%)$, essa etapa inicial geralmente é catalisada pelas amilases (ARAÚJO, 2012). Após essa primeira etapa chamada de liquefação ocorre a sacarificação, na qual ocorre a hidrólise completa para a obtenção da glicose. A designação do conteúdo de enzimas e a duração do tratamento enzimático para a etapa de sacarificação depende da finalidade imposta ao produto final, ou seja, ao tipo de hidrolisado que se objetiva obter (ARAÚJO, 2012).

\section{Hidrólise ácida}

Na hidrólise ácida, os compostos mais utilizados são os ácidos nítrico, clorídrico ou sulfúrico, os quais podem ser diluídos ou concentrados, com rendimento de aproximadamente 75 - 90\% de açúcares (LU et al., 2007). O ácido sulfúrico é o mais usado, em virtude de seu baixo custo e boa eficácia. A hidrólise ácida é dependente de altas temperaturas $\left(160-230^{\circ} \mathrm{C}\right)$, com a concentração de ácido entre $2-5 \%$. A principal vantagem da hidrólise ácida é tempo relativamente curto de sacarificação, no entanto como desvantagens aponta-se a ocorrência de corrosão dos equipamentos, bem como a necessidade de realizar um tratamento de neutralização na solução dos açúcares para fermentação (MENON; RAO, 2012).

A hidrólise ácida pode ser empregada como pré-tratamento e hidrólise ou conjuntamente com a hidrólise enzimática (SANTOS et al., 2012). A hidrólise ácida se enquadra no prétratamento químico dos materiais lignocelulósicos (resíduos) (ALVES, 2012). Segundo Odunfa; Olanbiwoninu (2012), para maximizar a produção do etanol por meio do material 
lignocelulósico da mandioca é importante que esses resíduos sejam submetidos ao prétratamento com ácido sulfúrico antecedendo a fermentação. O uso do ácido diluído é mais comum, pois o ácido concentrado requer equipamentos resistentes a corrosão aumentando assim os custos de produção (GURGEl, 2010; SILVA, 2017).

\section{Fermentação alcoólica}

O etanol pode ser obtido por meio da fermentação de resíduos de plantas, materiais lignocelulósicos e produtos agrícolas (WU, 2014; KUMAR et al., 2015). Na fermentação ocorre a conversão de glicídios a etanol e $\mathrm{CO}_{2}$ por meio da ação de microorganismos, como por exemplo leveduras e fungos (NELSON; COX, 2011). O principal agente biológico utilizado no processo de fermentação é a levedura Saccharomyces cerevisiae, uma característica relevante dessa levedura é a capacidade de fermentar tanto em ausência quanto na presença de oxigênio, no entanto, para a condição de presença de $\mathrm{O}_{2}$ requer altos índices de açúcares (ARAÚJO, 2012).

Além das leveduras do gênero Saccharomyces destacam-se também o gênero Kluyveromyces e a bactéria Zymomonas mobilis (HE et al., 2014; NURHAYATI et al., 2016). Dentre as espécies que compõem o gênero Saccharomyces, destacam-se a Saccharomyces cerevisiae e Saccharomyces uvarum (NILSON; COX, 2011).

\section{Destilação}

O etanol presente no conteúdo obtido na fermentação é recuperado pela destilação (OLIVEIRA et al., 2012), ocorre nessa etapa a separação do etanol aos demais produtos e da água da etapa anterior (fermentação). O meio fermentado é destilado através de etapas em colunas de destilação, as quais são distribuídas em quatro troncos: destilação propriamente dita, retificação, desidratação e recuperação do desidratante (OLIVEIRA et al., 2012; VEIGA, 2012).

O líquido que será destilado no processo de fabricação do etanol com a mandioca como matéria-prima, será o vinho ou mosto fermentado, onde essa mistura advinda da etapa da fermentação é aquecida até atingir a ebulição, no entanto os vapores são resfriados (FABRICIO, 2011).

\section{METODOLOGIA}

Este trabalho é uma pesquisa exploratória de natureza qualitativa, que utilizou dados principalmente de trabalhos cientificos publicados em periodicos nacionais e internaicionais, 
VIABILIDADE DA UTILIZAÇÃO DA MANDIOCA PARA PRODUÇÃO DE ETANOL

bem como dissertações e teses, realizando as buscas de forma digital utilizando o google acadêmico, Science Direct e SciELO. A pesquisa foi realizada entre os meses de junho e outubro de 2020 .

\section{RESULTADOS E DISCUSSÃO}

Perante analise feita na revisão de literatura, pode-se percerber que a cultura da mandioca apresenta grande potencialidade para a obtenção do etanol (VALLE et al., 2009; SRIROTH et al., 2010), por armazenar amido em suas raízes a mandioca requer processos para poder ser realizada a fermentação alcoólica (OLIVEIRA et al., 2012). De acordo com Cabello (2010), ainda há um amplo espaço para ser explorado em termos de produtividade para a cultura da mandioca, enquanto que para a cana-de-açúcar que é a matéria-prima mais utilizada, os custos de produção estão cada vez maiores e com menores incrementos de produtividade.

Além da sua alta potencialidade, o cultivo da mandioca para fins de produção de biocombustíveis apresenta vantagens em virtude do seu fácil cultivo, podendo ser cultivada em áreas que apresentam improdutividade para as demais culturas, com poucas exigências hídricas e nutricionais (CINELLI, 2012). Para aumentar e facilitar o uso da mandioca para diversos fins, a Empresa Brasileira de Pesquisa Agropecuária (EMBRAPA) tem buscado desenvolver pesquisas a favor da cultura (CINELLI, 2012). Segundo Ereno (2008), há relatos que pesquisadores da EMBRAPA em busca de espécies exóticas na Amazônia, encontraram uma variedade de mandioca com alto teor de açúcares em suas raízes, a maioria sendo glicose. O uso dessa mandioca açucarada para a produção do etanol eliminaria a etapa da hidrólise do amido, reduzindo custos e o fluxograma operacional.

O amido contido nas raízes tuberosas da mandioca é de grande importância para a produção do etanol. No entanto, os subprodutos do processamento da mandioca apresentam potencialidades para os biocombustíveis, incluindo resíduos que geralmente são empregados para alimentação animal, e que poderiam ser usados geração de etanol, obtendo aumento no valor agregado e oferecendo benefícios aos produtores (SILVA et al., 2018). O processamento da mandioca para a aquisição de farinhas e da fécula gera subprodutos líquidos, como a manipueira, e a sólidos como a casca, entrecasca, fibra, farelo e descarte. Esses subprodutos podem ocasionar efeitos maléficos ao meio ambiente, uma vez que podem liberar o cianeto. Portanto, há uma relevância em estudos que demonstrem alternativas para o uso desses produtos visando a sustentabilidade e maior rendimento econômico (PEIXOTO; RESCH, 2018).

A produção do etanol por meio da mandioca apresenta-se como uma alternativa rentável para produtores em menor escala, no entanto não há a pretensão de substituir a cultura da cana- 
de-açúcar, mas tornar-se uma opção para o período de entressafra da cana-de-açúcar, bem como em regiões onde essa cultura não se estabeleça de forma adequada (CARDONA; SANCHÉZ, 2007). Nisso consiste mais uma vantagem da cultura da mandioca para a finalidade da produção do etanol, a mandioca se adapta a diferentes regiões e possibilita cultivos consorciados, bem como fornece o aproveitamento de seus resíduos e subprodutos e a colheita ao longo do ano sem a necessidade de máquinas agrícolas para colher, reduzindo assim os custos de seu cultivo (PEDUZZI, 2009). Com isso, a mandioca destaca-se quando comparada a cereais e cana-deaçúcar devido seu potencial produtivo elevado em regiões tropicais com baixa pluviosidade e fertilidade do solo. Devido ao seu rendimento, viabilidade e balanço de massa na obtenção do etanol a mandioca apresenta potencialidade em regiões pouco desenvolvidas (VALLE et al., 2009).

Selvakumar et al. (2018) em seu estudo destacou o caule da mandioca como sendo uma fonte uma matéria-prima promissora para a produção do etanol, uma vez que o conteúdo de biomassa do caule consiste basicamente em lignina e carboidratos. Com o uso desse material lignocelulósico para obter o etanol, reduz a poluição e possíveis problemas ocasionados ao meio ambiente e a saúde humana, uma vez que normalmente esse material é desperdiçado e/ou queimado (NUWAMANYA et al., 2012). No processamento da mandioca são gerados aproximadamente 0,47 toneladas de resíduos, levando em consideração 1 tonelada de mandioca (MOSHI et al., 2015). Considerando que a glicose existente nesses resíduos de biomassa lignocelulósica fosse convertida em etanol, poderia produzir cerca de 2.576 litros de etanol/ha levando assim ao aproveitamento total desses materiais. Comparando com os resíduos da canade-açúcar (palha) onde a produção pode alcançar até 3.444 litros de etanol/ha, é necessário que haja um aperfeiçoamento tecnológico e mais pesquisas que estabeleçam uma maior produtividade utilizando esse material lignocelulósico (SANTOS et al., 2012). Visando maximizar o uso desses recursos, pode haver uma conversão biológica em diversos produtos considerados de alto valor (ZHANG et al., 2016).

Apesar das vantagens no reaproveitamento da biomassa lignocelulósica encontrada no caule da mandioca, há um impedimento acerca da desestruturação desses compostos, uma vez que essa biomassa apresenta grande recalcitrância, sendo resistente as enzimas da hidrólise (MOHAPATRA et al., 2017). Com isso, é necessário um pré-tratamento que pode ser físico ou químico (SELVAKUMAR et al., 2018).

Apesar de todas as vantagens acerca da cultura, as usinas de produção do etanol por meio do uso da mandioca apresentam características inferiores comparadas as de cana-deaçúcar, em relação a viabilidade técnica e econômica. Isso ocorre em virtude do menor 
VIABILIDADE DA UTILIZAÇÃO DA MANDIOCA PARA PRODUÇÃO DE ETANOL

investimento nas etapas de beneficiamento, e com isso há uma maior distribuição das usinas, contribuindo assim para o desenvolvimento socioeconômico das regiões (VALLE et al., 2009; AGUIAR, 2011). O processamento da mandioca para obtenção do etanol se iguala ao da canade-açúcar nas etapas de fermentação e destilação. No entanto, para a conversão do amido em glicose o método mais utilizado é a hidrólise enzimática, sendo considerado o mais eficiente (SALLA, 2010). Além disso, o processamento por via enzimática é mais seguro, uma vez que não gera poluentes, dispensando assim os possíveis custos para viabilizar esses resíduos e podendo reaproveita-los para fins de alimentação animal. Vale salientar que as enzimas utilizadas nesse processo de hidrólise encontram-se em abundância no mercado e com um valor considerado baixo (VALLE et al., 2009).

Curvelo-Santana et al. (2010) buscaram a otimização do processo de hidrólise do amido de mandioca com $\alpha$-amilase de A. niger e obtenção do álcool deste xarope. Utilizaram-se a concentração de amido entre 7 e 22 g.L $\mathrm{L}^{-1}$ e temperatura entre 30 e $59,1^{\circ} \mathrm{C}$. O tempo para uma boa atividade da hidrólise com uma total conversão (cerca de 80\%) foi de 20-200 minutos, juntamente com a temperatura de $55-59,1^{\circ} \mathrm{C}$ e as concentrações de amido entre 7,9-10 ou 20$22 \mathrm{~g} . \mathrm{L}^{-1}$. Assim, o emprego do hidrolisado de amido de mandioca na produção de álcool é viável e ele pode ser fermentado, elevando o rendimento da produção das indústrias sucroalcooleiras. Camili e Cabello (2012) analisaram o processo de produção de etanol por hidrólise enzimática a partir de raízes de mandioca in natura. Diante dos resultados encontrados, os autores concluíram que a polpa integral de mandioca se apresentou como promissora matéria-prima para produção de etanol.

Martinez (2016) em seu estudo produziu etanol a partir do farelo de mandioca de diferentes fecularias. Para o processo de obtenção do etanol as amostras foram submetidas a hidrólise enzimática, empregando-se a alfa-amilase e amiloglucosidases, sendo posteriormente fermentadas e destiladas. Esse autor constatou que o farelo da mandioca apresentou potencial para a produção de etanol, com alto teor de amido e respondendo eficientemente a hidrólise e sacarificação a partir de enzimas. Vítor (2018) em seu estudo avaliou a eficiência da hidrólise do amido de mandioca, com diferentes tempos de ações enzimáticas para a produção de etanol. Utilizou-se diferentes variedades de mandioca e tempos de hidrólise verificou que a variedade BRS Tapioqueira apresentou maior potencial para a produção do etanol e o tempo de hidrólise com $3 \mathrm{~h} \alpha$ amilase e 4,5h amiloglucosidase foi recomendado.

Cabral (2016) avaliou o potencial de resíduos de mandioca durante o processamento de fécula como matéria-prima para a produção de bioetanol. O autor concluiu que os resíduos de mandioca servem como substrato comercial para produção de etanol. Esse experimento 
apresentou praticidade sobre as condições ideais para aproveitamento dos resíduos, com a geração de energia "limpa", sendo de fundamental importância onde aumenta cotidianamente a preocupação econômica e ambiental. Behera e Ray (2015) objetivaram em seu estudo sacarificar a farinha de mandioca por hidrólise ácida e hidrólise enzimática, e conversão do açúcar resultante em etanol por fermentação com as células imobilizadas de Saccharomyces cerevisiae. Os resultados gerais obtidos neste estudo sugerem que a farinha de mandioca pode ser usada como matéria-prima para a produção de etanol por fermentação com células imobilizadas de S. cerevisiae. A eficiência de conversão de açúcar em etanol foi de 94,74\%.

Emeka et al. (2015) objetivaram utilizar o resíduo celulósico da mandioca para a produção de bioetanol E2G utilizando hidrólise ácida e enzimática. Um total de 32,4\% de álcool foi obtido, o que indica que os resíduos da mandioca realmente podem ser transformados em produtos químicos para uso como combustíveis, produtos bioquímicos, intermediários sintéticos etc. O rendimento do álcool obtido no estudo é baixo e a comparação com estudos relacionados mostrou que o rendimento é ligeiramente superior. A integração do etanol $2 \mathrm{G}$ com o $1 \mathrm{G}$ poderia facilitar a melhor utilização da biomassa e estabilizar relativamente a economia de produção. Septia et al. (2018) buscaram identificar a disponibilidade e as características da biomassa residual do caule da mandioca gigante como matéria-prima para produção do etanol. Os autores sugeriram que a biomassa residual do caule de M. esculenta pudesse ser usada como matéria-prima sustentável para a produção de etanol. Pothiraj et al. (2015) avaliaram a eficiência da liquefação microbiana e enzimática, e sacarificação de resíduos de mandioca para a produção econômica de etanol utilizando Zymomonas mobilis. Os resultados deste estudo indicaram que a sacarificação microbiana e fermentação de resíduos de mandioca envolvendo Z. mobilis podem ser usados para melhorar a produção de etanol a partir de resíduos de mandioca ou outros resíduos de amido para reduzir o custo de produção de etanol, reduzindo o tempo total do processo completo.

Fabrício (2011) em seu estudo buscou determinar os custos envolvidos na produção do etanol pelo método de custeio baseado em atividades durante a condução do seu cultivo e a produção do etanol em uma usina de pequeno porte. Observaram o custo de $\mathrm{R} \$ 2.283,56$ para o cultivo de 1 hectare da mandioca que produz por volta de 25 toneladas. Os autores constataram que a partir de 1 tonelada de mandioca foi possível obter 188 litros de etanol, onde o custo é de $\mathrm{R} \$ 0,53 /$ litro somando-se ao custo de processamento, o preço da produção de etanol por meio da mandioca é de R \$ 1,01. O custo de processamento da obtenção do etanol observado foi de $\mathrm{R} \$ 246,77$ para $3.000 \mathrm{Kg}$ de mandioca, considerando que $39 \%$ dos custos foram de mão-deobra, $36 \%$ de insumos, $14 \%$ de lenha, $7 \%$ de energia elétrica e 5\% de água. Além disso, 
realizou-se a comparação da produtividade da matéria-prima, onde 35 toneladas de mandioca rendem cerca de 6580 litros de etanol, enquanto que 80 toneladas de cana-de-açúcar rendem cerca de 4000 litros de etanol.

\section{CONCLUSÕES}

A viabilidade da utilização da mandioca para obtenção do etanol é alternativa considerável, tendo em vista as vantagens existentes nesse processo. A possibilidade do uso de seus resíduos também é de grande importância, pois proporciona o aproveitamento total da mandioca e evita a poluição por meio do descarte de alguns resíduos tóxicos. No entanto, para que haja uma expansão e melhor aproveitamento da mandioca como matéria-prima para a produção do etanol, é necessário a realização de pesquisas que ofereçam subsídios para a redução dos custos de produção, bem como maximizem a produtividade da mandioca.

\section{REFERÊNCIAS}

AGUIAR, E. B. Estudo da poda da mandioca (Manihot esculenta Crantz). Botucatu ,2011, 144 p. Tese (Agronomia-Agricultura). Universidade Estadual Paulista, Faculdade de Ciências Agronômicas, , 2011.

ALMEIDA, J.; FERREIRA FILHO, J. R. Mandioca: uma boa alternativa para Alimentação Animal. Bahia Agrícola, v. 7, n. 1, p.50-56, 2005.

ALVES, J. M. B.; MACRI, R. C. V. Etanol de segunda geração: estudo de materiais lignocelulósicos e aplicações da lignina, 2012.

ALVIRA, P.; TOMÁS-PEJÓ, E.; BALLESTEROS, M.; NEGRO, M. J. Pretreatment technologies for an efficient bioethanol production process based on enzymatic hydrolysis: a review. Bioresource Technology, v. 101, n. 13, p. 4851-4861, 2010.

ANDRÉ, T. B.; SANTOS, A. C. Uso de produtos da cultura da mandioca (Manihot) na produção animal. Enciclopédia Biosfera, Centro Científico Conhecer, v. 8, n. 15; p. 16221647, 2012.

ARAÚJO, L. L. Produção de álcool a partir de amido utilizando-se amilases recombinantes. Manaus, 2012, 68 p. Dissertação (Biotecnologia). Programa de Pós-Graduação em Biotecnologia, Universidade Federal do Amazonas, 2012.

ARREDONDO, H. I. V. Avaliação exergética e exergo-ambiental da produção de biocombustíveis. São Paulo, 2009, 235 p. Tese (Engenharia Mecânica). Escola Politécnica da Universidade de São Paulo, 2009.

ASGHER, M.; AHMAD, Z.; IQBAL, H. M. N. Alkali and enzymatic delignification of sugarcane bagasse to expose cellulose polymers for saccharification and bio-ethanol production. Industrial Crops and Products, v. 44, p. 488-495, 2013. 
BEHERA, S.; MOHANTY, R. C.; RAY, R. C. Batch ethanol production from cassava (Manihot esculenta Crantz.) flour using Saccharomyces cerevisiae cells immobilized in calcium alginate. Annals Microbiol., v. 65, p. 779-783, 2015.

BIOFUEL. Biofuel Chemistry: How they Burn?. Disponível em: <http://biofuel.org.uk/howdo-biofuels-burn.html> . Acesso em: 14 de Julho de 2020.

CABELlO, C. Produção de etanol da mandioca. Disponível em: < http://www.abam.com.br/artigos>. Acesso em: 14 de Julho de 2020.

CABRAL, Á. S. Tratamentos ácidos de hidrólise e avaliação de parâmetros na produção de etanol celulósico utilizando resíduos de mandioca (Manihot esculenta Crantz). Sâo José do Rio Preto, 2016, 157 p. Dissertação (Engenharia e Ciência de Alimentos) Programa de Pós Graduação em Engenharia e Ciência de Alimentos. Universidade Estadual Paulista, 2016.

CAMILI, E. A.; CABELLO, C. Produção de etanol a partir de polpa de mandioca. Energia Agricultura, v. 27, n. 2, p. 1-19, 2012.

CARDONA, C. A.; SÁNCHEZ, Ó. J. Trends in biotechnological production of fuel ethanol from different feedstocks. Bioresource Technology, v. 99, n. 13, p. 5270-5295, 2007.

CINELLI, B. A. Produção de etanol a partir da fermentação simultânea à hidrólise do amido granular de resíduo agroindustrial. Rio de Janeiro, 2012, 183 p. Dissertação (Engenharia Química) -Programa de Pós-Graduação em Engenharia Química, Universidade Federal do Rio de Janeiro, 2012.

CONAB - Companhia Nacional de Abastecimento. Câmara setorial da cadeia produtiva de mandioca e derivados. Disponível em: <http://www.conab.gov.br/OlalaCMS/uploads/arquivos/11_11_21_16_00_02_mandiocanove mbro2011.pdf>.Acesso: 10 jul 2020.

CUNHA, S. M; GUEDES, F. F.; JUNIOR, R. W.; PALMA, M. B. Produção de Bioetanol a partir do Bagaço de Mandioca Hidrolisado por Glucoamilase obtida por Fermentação em Estado Sólido.In: XVII Simpósio Nacional de Bioprocessos, Natal, RN Anais do XVII Simpósio Nacional de Bioprocessos, 2009.

CURVELO-SANTANA, J. C.; EHRHARDT, D. D.; TAMBOURGI, E. B. Otimização da produção de álcool de mandioca. Ciênc. Tecnol. Aliment., v. 30, n. 3, p. 613-617, 2010.

DIAS, M. O. S.; JUNQUEIRA, T. L.; CAVALETT, O.; PAVANELLO, L. G.; CUNHA, M. P.; JESUS, C. D. F.; BONOMI, A. Biorefineries for the production of first and second generation ethanol and electricity from sugarcane. Applied Energy, v. 109, p. 72-78, 2013.

EMEKA, E. E.; ELEMIKE, E. E.; OSEGHALE, O. C.; OKOYE, A. C. Utilization of cellulosic cassava waste for bio-ethanol production. Journal of Environmental Chemical Engineering, v. 3, n. 4, p. 2797-2800, 2015.

ERENO, D.; Site Inovação Tecnológica. Bioetanol pode ser produzido de nova variedade de mandioca doce. 12/03/2008. [On-line]. Disponível em: $<w w w$. inovacaotecnologica.com.br/noticias/artigo=bioetanol-pode-ser-produzido-70 denovavariedade-de-mandioca-doce>. Acesso em: 22 de Julho de 2020. 
FABRICIO, A. M. Determinaçao dos custos de produçao do etanol a partir da mandioca (Manihot esculenta Crantz) pelo método de custeio baseado em atividades (ABC). Santa Maria, 2011, 137 p. Dissertação (Engenharia de Produção). Universidade Federal de Santa Maria, 2011.

FAO. 2019. Food and Agriculture Organization of the United Nations - FAOSTAT. 2019. Production, crops. Disponível em: <http://www.fao.org/faostat/en/\#home/>. Acesso em: 07 de Agosto de 2020.

FAO. 2020. Crops. Disponível em: < http://www.fao.org/faostat/en/\#data/QC>. Acesso em: 07 de Julho de 2020.

FAO. 2020. Food and Agriculture Organization of the United Nations - FAO. Save and grow: cassava. A guide to sustainable production intensification. 2013. Disponível em: <http://www.fao.org/ag/save-and-grow/cassava/pt/index.html >. Acesso em: 07 de Agosto de 2020.

FAOSTAT. 2019. Food and Agriculture Organization of the United Nations. Production. Disponível em: <http://www.fao.org/faostat/en/\#data>. Acesso em: 14 outubro de 2020.

FENNEMA, O. R.; KIRK L. P.; SRINIVASAN, D, Química de alimentos de Fennema, Traduzido por Adriano Brandelli, Ed. Artmed, 4ª ed. Porto Alegre, 2010.

FERREIRA, A. L.; SILVA, A. F.; PEREIRA, L. G. R.; BRAGA, L. G.; MORAES, S.A.; ARAÚJO, G. G. L. Produção e valor nutritivo da parte aérea da mandioca, maniçoba e pornúncia. Rev. Bras. Saúde, v. 10, n. 1, p. 983-990, 2009.

FIORDA, F. A.; SOARES JÚNIOR, M. S.; SILVA, F. A. D.; SOUTO, L. R. F.; GROSSMANN, M. V. E. Farinha de bagaço de mandioca: aproveitamento de subproduto e comparação com fécula de mandioca. Pesquisa Agropecuária Tropical, v. 43, n. 4, p. 408416, 2013.

GURGEL, L.V. Hidrólise ácida de bagaço de cana-de-açúcar: estudo cinético de sacarificação de celulose para a produção de etanol. São Carlos, 2010, 315 p. Tese (Ciências). Instituto de Química de São Carlos da Universidade de São Paulo, 2010.

HE, M. X.; WU, B.; QIN, H.; RUAN, Z. Y.; TAN, F. R.; WANG, J. L. ... TANG, X. Y. Zymomonas mobilis: a novel platform for future biorefineries. Biotechnology for Biofuels, $\mathrm{v}$. 7, n. 1, p. 101, 2014.

IEA. 2017. Tracking Clean Energy Progress. Paris: OECD. Acesso em : <https://www.iea.org/publications/freepublications/publication/TrackingCleanEnergyPro gress2017.pdf $>$. 14 de outubro 2020.

IQBAL, H. M. N.; KYAZZE, G.; KESHAVARZ, T. Advances in the valorization of lignocellulosic materials by biotechnology: an overview. BioResources, v. 8, n. 2, p. 31573176, 2013.

IYER, S.; MATTINSON, D. S.; FELLMAN, J. K.Study of the early events leading to cassava root postharvest deterioration. Tropical Plant Biology, v. 3, p. 151-165, 2010.

KUMAR, U.; ARORA, A. S.; PARIKH S. Sustainable synthesis of clean energy fuel Bio- 
ethanol from low cost biomass resources. International Journal of Advance Research in. Engineering, Science \& Technology, v. 2, p. 2394-2444, 2015.

LACERDA, L. G.; ALMEIDA, R. R.; DEMIATE, I. M.; CARVALHO FILHO, M. A. S.; VASCONCELOS, E. C.; WOICIECHOWSKI, A. L. ... \& SOCCOL, C. R. Thermoanalytical and starch content evaluation of cassava bagasse as agro-industrial residue. Brazilian Archives of Biology and Technology, v. 52, p. 143-150, 2009.

LEONEl, M.; CEREDA, M. P. Avaliação da concentração de pectinase no processo de hidrólise-sacarificação do farelo de mandioca para obtenção de etanol. Ciência e Tecnologia de Alimentos, v. 20, n. 2, p. 220-227, 2000.

LIMA, A. L. A. Biorreatores utilizados na hidrólise enzimática para produção de etanol de segunda geração. Lorena, 2013, 46 p. Monografia (Engenharia Bioquímica). Escola de Engenharia de Lorena da Universidade de São Paulo, 2013.

LIMA, G. F. N. Fermentação alcoólica do hidrolisado de manipueira. João Pessoa, 2017, 41 p. Monografia (Tecnologia e Produção Sucroalcooleira). Centro de Tecnologia e Desenvolvimento Regional, Universidade Federal da Paraíba, 2017.

LOUREIRO, A. C.; SOARES, J. V. S.; SILVA CHAAR, J.; FREITAS HIDALGO, A.; SOUZA, L. D. S. S.; PEREIRA, A. M. Avaliação do potencial dos resíduos (casca, entrecasca e pontas) do beneficiamento da mandioca (Manihot esculenta Crantz) para a produção de bioetanol utilizando hidrólise ácida. Brazilian Applied Science Review, v. 4, n. 2, p. 606-620, 2020.

LU, X. B.; ZHANG, Y. M.; YANG, J.; LIANG, Y. Enzymatic hydrolysis of corn stover after pretreatment with dilute sulfuric acid. Chemical Engineering \& Technology, v. 30, n. 7, p. 938-944, 2007.

MARTINEZ, D. G.; FEIDEN, A. Potencial do resíduo do processamento da mandioca para produção de etanol de segunda geração. Revista Brasileira de Energias Renováveis, v. 6, n. 2, p. 141-148, 2017.

MARTINEZ, D. G. Produção do etanol de segunda geração a partir de resíduos do processamento da mandioca. Cascavel, 2016, 47 p. Dissertação (Energia na Agricultura). Universidade Federal Oeste do Paraná, 2016.

MDA - Ministério do Desenvolvimento Agrário. Disponível em: <http://siater.mda.gov.br/〉. Acesso em 10 jul 2020.

MENON, V.; RAO, M. Trends in bioconversion of lignocelluloses: Biofuels, platform chemical e biorefinary concept. Prog. En. Comb. Sci., v. 38, n. 4, p. 522-550, 2012.

MOHAPATRA, S.; DANDAPAT, S. J.; THATOI, H. Physicochemical characterization, modeling and optimization of ultrasono-assisted acid pretreatment of two Pennisetum sp. using taguchi and artificial neural networking for enhanced delignification. J. Environ. Manag., v. 187, p. 537-549, 2017.

MOSHI, A. P.; TEMU, S. G.; NGES, I. A.; MALMO, G.; HOSEA, K. M.; ELISANTE, E.; MATTIASSON, B. Combined production of bioethanol and biogas from peels of wild cassava Manihot glaziovii. Chemical Engineering Journal, v. 279, p. 297-306, 2015. 
MÜLLER, G. Clonagem e expressão do gene da alfa-amilase de Bacillus subtilis variedade D2 na levedura Pichia pastoris. Manaus, 2008, 108 p. Dissertação ( Genética, Conservação e Biologia Evolutiva), Universidade Federal do Amazonas, 2008.

NELSON, D. L.; COX, M. M.; LEHNINGER, A. L. Princípio de bioquímica. Traduzido por SIMÕES, A. A.; LODE, W. R. N. São Paulo: Ed. SARVIER, 5a ed., 839p., 2011.

NURHAYATI, C. L. C.; Dillirani Nagarajan, J. S. C. Immobilization of Zymomonas mobilis with $\mathrm{Fe}_{2} \mathrm{O}_{3}$-modified polyvinyl alcohol for continuous ethanol fermentation. Biochem. Eng. J., v. 114, n. 1, p. 298-306, 2016.

NUWAMANYA, E.; KARLTUN, C. L.; KAWUKI, R. S.; BAGUMA, Y. Bioethanol production from non-food parts of Cassava (Manihot esculenta Crantz). Ambio, v. 41, p. 262$270,2012$.

OCDE/FAO - Organização para a cooperação e desenvolvimento econômico. Organização das Nações Unidas para Agricultura e Alimentação. Perspectivas Agrícolas 2016-2025, OECD Publishing, París. 2016. Disponível em: < http://www.fao.org/3/a-i5778s.pdf>. Acesso em: 14 de Julho de 2020.

ODUNFA, S. A.; OLABIWONINU, A. A. Enhancing the production of reducing sugars from cassava peels by pretreatment methods. International J. Sci. Technol., v. 2, n. 9, p. 650-657, 2012.

OLIVEIRA, L. M.; SERRA, J. C. V.; MAGALHÃES, K. B. Estudo comparativo das diferentes tecnologias utilizadas para produção de etanol. Geoambiente on-line, n. 19, p. 1-23., 2012.

PANDEY, A.; WEBB, C.; SOCCOL, C. R.; LARROCHE, C. Enzyme Technology. New Delhi: Asiatech Publishers, 1 ed., Inc, p. 760, 2005.

PEDUZZI, P. Produção de etanol a partir da mandioca pode ser mais barato que cana. Agência Brasil. 2009. Disponível em < http://www.jornaldaciencia.org.br/Detalhe.jsp?id=61939>. Aceso em 20 de Julho de 2020.

PEIXOTO, T. S.; RESCH, S. Resíduos de mandioca: um estudo sobre a destinação da massa de mandioca pelas fecularias brasileiras. In: Encontro Internacional de Gestão, Desenvolvimento e Inovação (EIGEDIN), v. 2, n. 1, 2018.

PEREIRA, S. C.; MAEHARA, L.; MACHADO, C. M. M.; FARINAS, C. S. Physico-chemicalmorphological characterization of all lignocellulosic biomass from sugar cane used for the production of $2 \mathrm{G}$ ethanol by spectroscopy and microscopy techniques. Renewable Energy, v. 87, p. 607-617, 2016.

POTHIRAJ, C.; ARUN, A.; EYINI, M. Simultaneous saccharification and fermentation of cassava waste for ethanol production. Biofuels Res. J., v. 2, p. 196-202, 2015.

RABELO, S. C.; FONSECA, N. A.; ANDRADE, R. R.; MACIEL FILHO, R.; COSTA, A. C. Ethanol production from enzymatic hydrolysis of sugarcane bagasse pretreated with lime and alkaline hydrogen peroxide. Biomass and Bioenergy, v. 35, n. 7, p. 2600-2607, 2011.

RFA - RENEWABLE FUELS ASSOCIATION. Fueling a high octane future: 2016 Ethanol Industry Outlook. Disponível em: < https://ethanolrfa.org/wp- 
content/uploads/2016/02/Ethanol-Industry-Outlook-2016.pdf >. Acesso em: 10 de Julho de 2020.

RIBEIRO, D. M. L. Caracterização e comportamento sacarificante da flora microbiana empregada na fabricação da aguardente de mandioca (Tiquira). São Luís, 2011, 80 p. Dissertação (Química). Universidade Federal do Maranhão. 2011.

SALLA, D. A.; FURLANETO, F. P.; CABELLO, C.; KANTHACK, R. A. Análise energética de sistemas de produção de etanol de mandioca (Manihot esculenta Crantz). Revista Brasileira de Engenharia Agrícola e Ambiental, v. 14, n. 4, p. 444-448, 2010.

SANTOS, F. A.; QUEIRÓZ, J. H. D.; COLODETTE, J. L.; FERNANDES, S. A.; GUIMARÃES, V. M.; REZENDE, S. T. Potencial da palha de cana-de-açúcar para produção de etanol. Química nova, v. 35, n. 5, p. 1004-1010, 2012.

SELVAKUMAR, P.; KAVITHA, S.; SIVASHANMUGAM, P. Optimization of process parameters for efficient bioconversion of thermo-chemo pretreated Manihot esculenta Crantz YTP1 stem to ethanol. Waste and Biomass Valorization, v. 10, n. 8, p. 2177-2191, 2019.

SEPTIA, E; SUPRIADI; SUWINARTI, W; AMIRTA, R. Characterization and ethanol potential from giant cassava (Manihot esculenta) stem waste biomass. Iop Conference Series: Earth and Environmental Science, [S.L.], v. 144, p.1-8, 2018.

SHAHZADI, T.; MEHMOOD, S.; IRSHAD, M.; ANWAR, Z.; AFROZ, A.; ZEESHAN, N. ... \& SUGHRA, K. Advances in lignocellulosic biotechnology: A brief review on lignocellulosic biomass and cellulases. Advances in Bioscience and Biotechnology, v. 5, 246-251, 2014.

SILVA, F. V. Panorama e perspectivas do etanol lignocelulósico. Revista Liberato, v. 13, n. 20, p. 1-16, 2012.

SILVA, I. B. Potencialidades energéticas proveniente dos resíduos da mandioca (Manihot esculenta Crantz). João Pessoa, 2017, 65 p. Dissertação (Energias Renováveis). Universidade Federal da Paraíba, 2017.

SILVA, R. C. C. Subprodutos da mandioca (Manihot esculenta Crantz) na alimentação de codornas. Rio Largo 2018, 39 p. Monografia (Zootecnia). Universidade Federal de Alagoas, 2018.

SILVA, V. F. N. Estudo do pré-tratamento e sacarificação dos resíduos agríco-industriais como etapas no processo de obtenção do etanol celulósico. Lorena, 2009, 116 p. Dissertação (Biotecnologia Industrial). Universidade de São Paulo, 2009.

SORDA, G.; BANSE, M.; KEMFERT, C. An overview of biofuel policies across the world. Energy Policy, v. 38, n. 11, p. 6977-6988, 2010.

SUBASHINI, D.; EJILANE, J.; RADHA, A.; JAYASRI, M. A.; SUTHINDHIRAN, K. Ethanol Production from Sago Waste Using Saccharomyces cerevisiae Vits-M1.Cur. Res. J. Biol. Sci., v. 3, n. 1, p. 42-51, 2011.

SUMAN, P. A.; URBANO, L. H.; LEONEL, M.; MISCHAN, M. M. Efeitos de parâmetros de fermentação na produção de etanol a partir de resíduo líquido da industrialização da mandioca (manipueira). Revista Acta Scientiarum. Technology, v. 33, n. 4, p. 379-384, 2011. 
TAYLOR, M. P.; ELEY, K. L.; MARTIN, S.; TUFFIN, M. I.; BURTON, S. G.; COWAN, D. A. Thermophilic ethanologenesis: future prospects for second-generation bioethanol production. Trends in Biotechnology, v. 27, n. 7, p. 398-405, 2009.

USDA. China - Peoples Republic of Biofuels Annual. Global Agricultural Information Network. Annual Report. p. 1-12, 2011.

VALLE, T. L.; FELTRAN, J. C.; CARVALHO, C. R. L. Mandioca para a produção de etanol. Infobibos. $2009 . \quad$ Disponível em: <http://www.infobibos.com/Artigos/2009_4/mandioca/index.htm>. Acesso em: 31 de Julho de 2020.

VALLE, T.L.; LORENZI, J. O. Variedades melhoradas de mandioca como instrumento de inovação, segurança alimentar, competitividade e sustentabilidade: contribuições do Instituto Agronômico de Campinas (IAC). Caderno de Ciência \& Tecnologia, v. 31, p. 15-34, 2014.

VÍTOR, L. A. D. A eficiência da hidrólise do amido de mandioca (Manihot esculenta Crantz), em diferentes tempos de ações enzimáticas, visando a produção de etanol. Palmas, 2018, 50 p. Monografia (Agroenergia). Universidade Federal do Tocantins. 2018.

ZAMORA-HERNÁNDEZ, T.; PRADO-FUENTES, A.; CAPATAZ-TAFUR, J.; BARRERAFIGUEROA, B. E.; PEÑA-CASTRO, J. M. Demostraciones prácticas de los retos y oportunidades de la producción de bioetanol de primera y segunda generación a partir de cultivos tropicales. Educación Química, v. 25, p. 265-274, 2014.

ZHANG, M.; XIE, L.; YIN, Z.; KHANAL, S. K.; ZHOU, Q. Biorefinery approach for cassavabased industrial wastes: current status and opportunities. Bioresource Technology, v. 215, p. 50-62, 2016. 